PHYSICAL ACTIVITY LEVELS ACCORDING TO GOLD GROUPING IN PATIENTS WITH COPD

Jl Canavan, SSC Kon, CM Nolan, SE Jones, MI Polkey, WD-C Man; NIHR Respiratory Biomedical Research Unit \& Imperial College London, Harefield, Middlesex, UK

\subsection{6/thoraxjnl-2013-204457.186}

Background The updated Global Initiative for Chronic Obstructive Lung Disease (GOLD) guidelines recognise the limitations of using grading systems based on spirometry alone. Understanding the impact of COPD on an individual patient should combine symptomatic assessment (using MRC Dyspnoea scale or COPD Assessment Test), spirometric classification and/or risk of
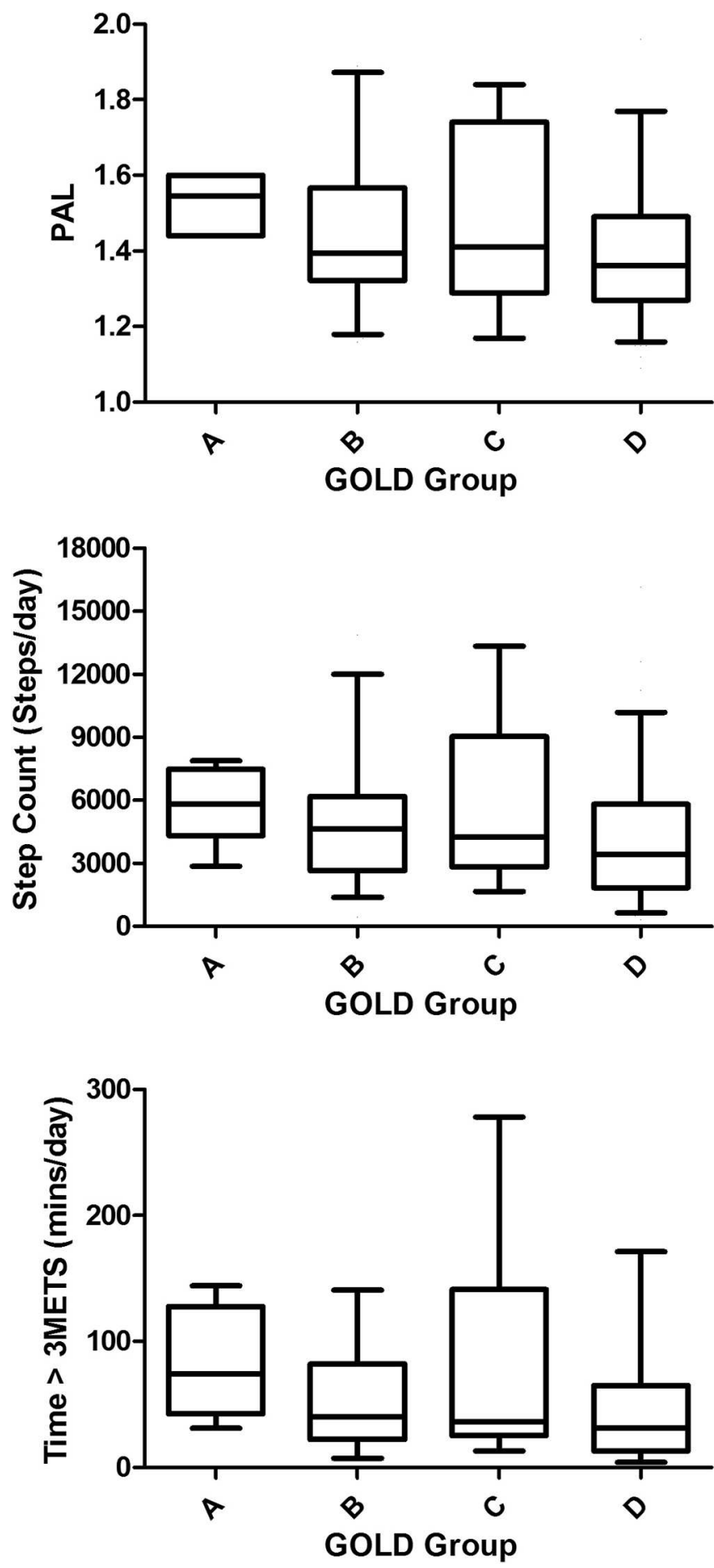

Abstract P36 Figure 1. exacerbations. GOLD recommends classifying patients into four groups: A (Low Risk, Less Symptoms), B (Low Risk, More Symptoms), C (High Risk, Less Symptoms) and D (High Risk, More Symptoms). Physical inactivity level is an important predictor for hospital admissions and mortality in patients with COPD. The aim of the study was to document objective physical activity parameters according to GOLD grouping. We hypothesised that physical activity levels would be highest in Group A and lowest in Group D.

Methods 220 patients with COPD (mean age 69 years, 81F:139M, mean FEV1 51\%predicted) were classified into GOLD groups A-D. Physical activity (PA) was measured for seven days using an accelerometer (Sensewear ${ }^{\circledR}$ ) as previously described (Watz et al., 2009). Physical activity parameters were analysed by researcher blinded to GOLD grouping, and included physical activity level (PAL: Total energy expenditure / resting energy expenditure), step count (steps per day) and time spent in at least moderate activity, defined as greater than 3 METS. Kruskal-Wallis was used to compare physical activity parameters across groups and Dunn's multiple comparison post-hoc test to compare between individual groups.

Results Parameters of physical activity according to GOLD group are shown in Figure 1. Although multiple group comparison showed significant differences in physical activity (PAL: $p=0.04$, Step Count: $p=0.005$, 3METS: $p=0.02$ ), post-hoc tests revealed no significant differences in PAL or 3METS between individual groups. For step count, there was a significant difference in rank sum between groups $\mathrm{B}$ and $\mathrm{D}(\mathrm{p}<0.05)$, but not between any other individual group comparisons.

Conclusions There are complex and multifactorial determinants of physical activity levels in patients with COPD which cannot be distinguished by new GOLD grouping.

\section{P37 CHANGES IN DAILY PHYSICAL ACTIVITY IN COPD}

${ }^{1}$ AM Albarrati, ${ }^{1}$ NS Gale, ${ }^{2} S$ Enright, ${ }^{1}$ IC Munnery, ${ }^{1} M M$ Munnery, ${ }^{1} J R$ Cockcroft, ${ }^{1} D J$ Shale; ${ }^{1}$ Cardiorespiratory Medicine, Cardiff University, Cardiff, United Kingdom; ${ }^{2}$ Physiotherapy Department, Cardiff University, Cardiff, United Kingdom

\subsection{6/thoraxjnl-2013-204457.187}

Background COPD is associated with extrapulmonary manifestations that worsen patient's daily physical activity (DPA)(1). However, changes in DPA over time and contributing factors in this population have not been established. We hypothesise that patients with COPD would have lower DPA after one year.

Method As part of a longitudinal study in COPD (ARCADE), DPA was recorded as the number of steps using a multisensor armband (SenseWear Pro armband) in 28 patients with stable COPD for 7 days and repeated after one year for a further 7 days. Spirometry, body composition, handgrip strength (HGS), six-minute walk distance (6MWD), the COPD assessment test (CAT) were also recorded.

Results Patients mean (SD) age was 69 (7) years, BMI 27.2 (4.7) Kg/ $\mathrm{m}^{2}$ and $\mathrm{FEV}_{1} \%$ predicted $52(15)$. After one year, there was a reduction in the number of daily steps by 498 (218) steps and fat free mass (FFM) $1.10(1.75) \mathrm{kg}$, with an increase in fat mass (FM) 1.06 (2.24) $\mathrm{kg}$ and FFM/FM 0.11 (01), all $\mathrm{p}<0.01$. No changes were observed in $\mathrm{FEV}_{1} \%$, BMI, HGSor 6MWD. The rate of decline in the number of steps was $12.3 \%$ per year which related to baseline FFM, $\mathrm{r}=$ 0.51 and HGS, $\mathrm{r}=-0.53$, all $\mathrm{p}<0.01$. Linear regression analysis showed that FFM and HGS can predict $23 \%$ and $26 \%$ respectively of the variability in the reduction in the number of steps.

Conclusion There was a significant reduction in daily physical activity after one year, which related to baseline FFM and HGS, but was not associated with lung function and 6MWD. Despite 
maintaining the BMI level, there was a fat free mass turnover, which may indicate a hidden muscle catabolism. Therefore, early management to prevent muscle mass loss would improve patient's daily physical activity and quality of health.

\section{REFERENCES}

1. Watz, K et al (2008) AJRCCM 177(7), pp.743-751.

\section{P38 IS IT POSSIBLE TO PREDICT AMBULATORY OXYGEN (AO) REQUIREMENTS?}

${ }^{1} \mathrm{~L}$ Cornish, ${ }^{1} \mathrm{~F}$ Dyer, ${ }^{2} \mathrm{~K}$ Cheema, ${ }^{1} \mathrm{~J}$ Bott; ${ }^{1}$ Virgin Care Respiratory Care Team, Chertsey, United Kingdom; ${ }^{2}$ South East Coast Quality Observatory, Horley, united kingdom

\subsection{6/thoraxjnl-2013-204457.188}

Aim To determine whether $\mathrm{AO}$ flow rate requirements can be predicted in patients with chronic lung diseas.

Background The process for AO assessment as per current UK guidance can be extremely time consuming, necessitating multiple walking tests with significant rest periods between walks. We set out to explore whether a model could accurately predict the $\mathrm{AO}$ flow rate required to abolish/minimise desaturation, thus simplifying the process.

Method Retrospectivedatawere analysed for all patients who attended AO clinic from April 2009 to January 2013. AO needs were assessed as per current UK guidance. For those who met the criteria for AO $(\mathrm{n}=100)$, resting Sp02, desaturation on the endurance shuttle walk test (ESWT) and AO flow rate required to minimise this desaturation were recorded, along with age and diagnosis. Results Multiple regression analysis was undertaken to test how well the variables included in the data could predict the likely level of $\mathrm{AO}$ required. A range of models were constructed and tested against the actual AO flow rate that had been required. The model which accounted for the greatest proportion of the variance $(\mathrm{r}=0.671, \mathrm{~F}=79.40, \mathrm{p}<0.05)$ included only one variable, the level of oxygen desaturation on room air post ESWT. Adding additional variable to the model, such as age and resting SpO2 did not add significantly to the predictive power of the model.

Conclusion Using this model, 91\% of patients were predicted correctly with an error of $+/-1 \mathrm{~L}$ of the actual flow rate. It would therefore, 9 times out of 10 , give a flow rate that was either correct $(+/-1 \mathrm{~L})$. Use of this model will reduce the number of walking tests required when performing $\mathrm{AO}$ assessment, saving both time and valuable healthcare resources.

\section{P39 A COMPARISON OF THE REPEATABILITY AND RESPONSIVENESS OF FIELD AND LABORATORY INCREMENTAL EXERCISE TESTS BETWEEN COPD AND CHRONIC HEART FAILURE}

TC Harvey-Dunstan, SJ Singh, MC Steiner, MDL Morgan, RA Evans; Department of Respiratory Medicine, Glenfield Hospital, Leicester, UK

\subsection{6/thoraxjnl-2013-204457.189}

Aim To describe and compare the repeatability and responsiveness of the Incremental Shuttle Walk Test (ISWT) and incremental cardiopulmonary exercise test between COPD and Chronic Heart Failure (CHF).

Hypothesis The null hypothesis, no difference in the measurement properties of ISWT and ICE between two chronic diseases. Methods Patients with symptomatic COPD and CHF (MRC 2 or NHYA II and above, respectively) were recruited. All patients underwent seven weeks of PR (1). At baseline, participants performed a familiarisation ISWT, two ISWTs and two incremental,
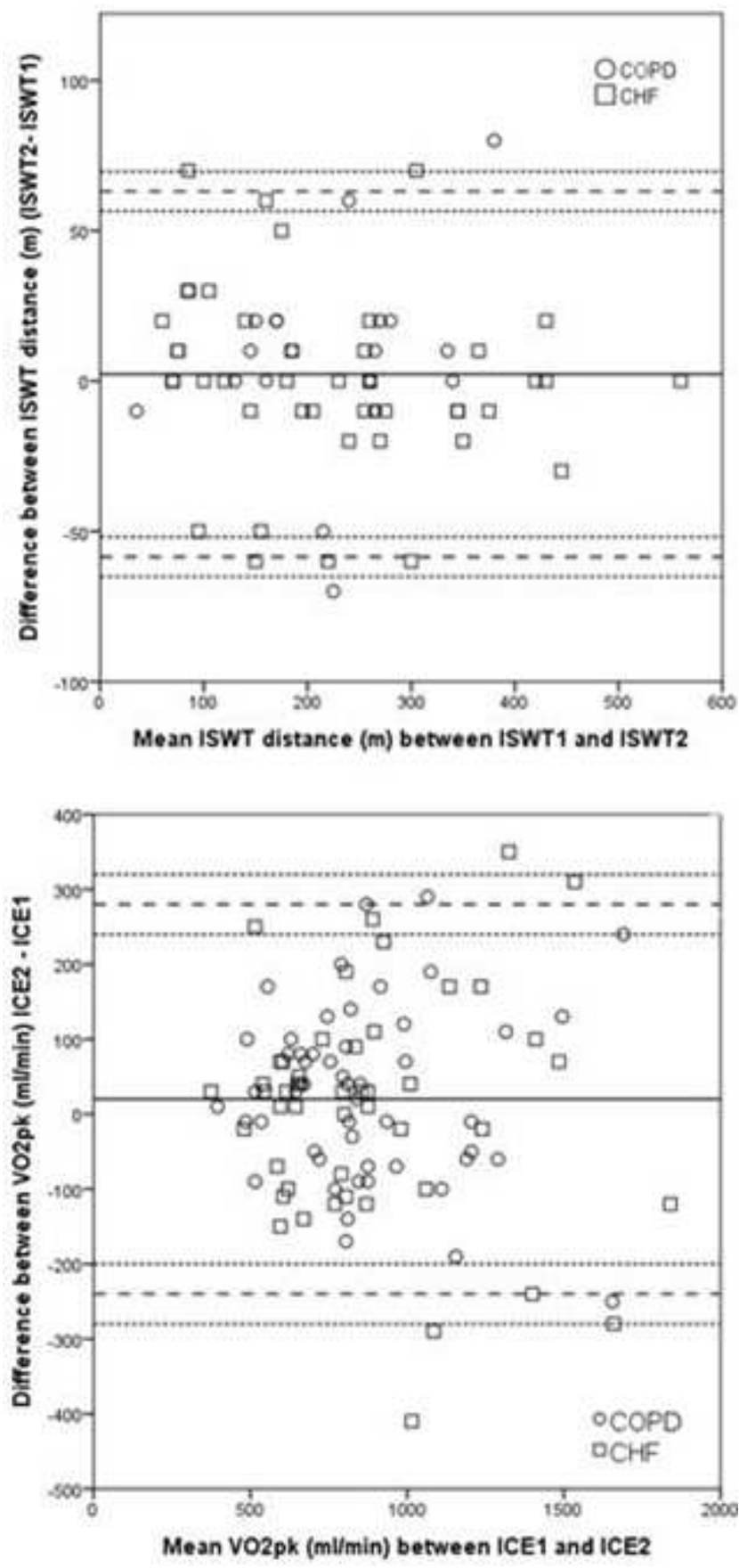

Abstract P39 Figure 1 repeatability of the ISWT distance and ICF VO2pk in COPD and CHF

symptom-limited, cardiopulmonary exercise tests on a cycle ergometer (ICE) within two weeks, on separate days. Both tests were repeated after seven weeks of PR.

Results 55 patients with COPD (70\% male, mean [SD] age 73 [9] yr, $\mathrm{FEV}_{1} \%$ predicted 43 [15], $\mathrm{FEV}_{1} / \mathrm{FVC} 0$ [9]) and 44 patients with CHF (66\% male, age 71 [11] yr, LVEF 33 [10]\%) were recruited.

There was a significant increase mean [SE] of 20 [4] m between the familiarisation ISWT and ISWT1 $(\mathrm{p}<0.001)$. There was no difference between either, ISWT1 and ISWT2, 2 (4) $\mathrm{m}(p=0.10)$, or peak oxygen uptake $\left(\mathrm{VO}_{2} \mathrm{pk}\right)$ for ICE1 and ICE2, $20[10] \mathrm{ml} \cdot \mathrm{min}^{-1}(p=0.16)$. There was no effect of disease, $\mathrm{p}=0.11$ and $\mathrm{p}=0.47$, respectively. Figure 1 shows the repeatability for the ISWT and ICE $\mathrm{VO}_{\mathrm{V} 2 \mathrm{pk}}$ in both conditions.

Mean [95\%CI] change in ISWT after PR was 68 [50-95] m, effect size $(E S) 0.58(\mathrm{p}<0.001)$ and $62[35-89] \mathrm{m}$, effect size 